Case Report

\title{
A Simplified Approach to Semi-Precision Attachment
}

\section{Krishna Prasad D. ${ }^{1}$, Archana Ashok Swaminathan ${ }^{2}$, Anupama Prasad D. ${ }^{3}$}

${ }^{1}$ Professor, ${ }^{2}$ Former Post Graduate student, ${ }^{3}$ Reader, Department of Prosthodontics and Crown \& Bridge, A.B. Shetty M emorial Institute of Dental Sciences, Nitte University, Deralakatte, M angalore -575018

*Corresponding Author: Dr. Krishna Prasad D., Professor,Department of Prosthodontics, A.B. Shetty M emorial Institute of Dental Sciences, NitteUniversity, Deralakatte, Mangalore-575018. E-mail: drkrishnaprasadd@yahoo.in.

Received

: 02-03-2015

Review Completed : 05-07-2016

Accepted

: 09-07-2016

Keywords : Semi-precision attachments, Sagix attachment, bar attachments, Precision attachments.

\begin{tabular}{|c|}
\hline Access this article online \\
\hline Quick Response Code \\
\hline
\end{tabular}

\begin{abstract}
:
Precision attachments open a new horizon of possibilities in prosthodontic rehabilitation. Varieties in size, shape and configuration allows them to be utilized in many clinical scenarios. However, the complex prosthesis with attachments does not serve the purpose of retention and esthetics but makes it cumbersome for the patient. Two cases in which a simplified approach to semi-precision attachments have been adopted, are discussed here.
\end{abstract}

\section{Introduction}

Precision attachments offer considerable advantages because of their flexibility. Nevertheless they have been ignored in the past largely due to notable cost and an inadequate grasp of their application. The last decade has seen the public become better informed and hence, a dental surgeon who familiarizes himself with precision attachments will add a new dimension to his treatment Options.[1]

An attachment is a connector consisting of two or more parts. One part is connected to a root, tooth or implant and the other part to prosthesis. (GPT 8) There are certain exceptions as some attachments have only one manufactured part and the other part must be made by the dentist or dental technician

There are two types of attachments:

1. Precision Attachments

2. Semi-Precision Attachment

\section{Precision versus Semi-Precision Attachments}

A precision attachment is fabricated from milled alloys and tolerances are within $.01 \mathrm{~mm}$. They are generally intracoronal and non-resilient. Their advantages include consistent quality, controlled wear and easier repair. They have standard parts which are interchangeable.[2]

A Semi-Precision attachment is fabricated by the direct casting of plastic, wax, or refractory patterns. They are considered "semi-precision" since in their fabrication they are subject to inconsistent water/powder ratios, burn out temperatures and other variables. The resulting components therefore, vary to a small degree. They are less costly, easy to fabricate and may be cast in alloy. They are generally extra coronal and resilient.

Where is it indicated?

- Aestheticszone

- Redistribution of forces required

- Minimize trauma to soft tissue 
- Control of loading and rotational forces

- Non parallel abutments present

- Segmenting of the long span bridge

- Future salvage efforts

- Improved retention

\section{Selection of Attachments}

There are a few criteria that help to decide the appropriate attachment based on the individual need of the case.

\section{Location 2. Function 3. Retention}

4. Space 5. Cost

Based on location, the semi precision attachments are divided into:-

Intracoronal Attachments: Placed within the contours of the crown form. The advantage of an intracoronal attachment is that the occlusal forces exerted upon the abutment tooth are applied close to the long axis of the tooth.

An intracoronal attachment however, usually requires a box preparation to allow the attachment to fit within the crown contour. If it is not possible to create a box preparation that will totally incorporate the female element, then an extracoronal attachment should be considered.Since all intracoronal attachments are nonresilient, double abutting is recommended.[3]

Extracoronal Attachments :Extracoronal attachments are positioned entirely outside the crown contour.

Advantages of extracoronal attachments are that the normal tooth contour can be maintained, minimal tooth reduction is necessary and the possibility of devitalizing the tooth is reduced. Also, the path of insertion is easier for patients with dexterity problems.

Most extracoronal attachments have some type of resiliency (stress redirectors). Even with resilient attachments, double abutting is recommended whenever possible.

It is however, more difficult to maintain hygiene with extracoronal attachments and patients should be instructed on the use of dental floss and hygiene accessories. This will help prevent unnecessary tissue irritation caused by food entrapment or calculus build-up.

\section{Radicular / Intraradicular Stud Type Attachments :} Radicular and Intraradicular stud type attachments are connected to a root preparation. The female or male is soldered or cast to a root cap coping .

The female element of intraradicular stud type attachments fit within the root form contour. The SwissLogic, Zest and the ZAAG are examples of this type of attachment.Some stud type attachments, such as the UniAnchor and the Direct 0-Ring, are directly cemented into the prepared root without requiring a cast coping. Stud type titanium implant attachments are also available to screw directly into implants or tissue extensions.

Bar Type: Bar type attachments span an edentulous area and connect abutment teeth, roots or implant. The removable bridge, partial denture, or over denture fits over the bar and is connected to it with one or more retention sleeves, riders/clips, or retentive plungers.

Based on function, it is important to differentiate between a solid and resilient type restoration. Abutment/tooth supported restorations are considered solid, where abutment/tooth and tissue supported restorations are considered resilient. Abutment/tooth supported attachments are sub classified into a two types, nonlockable and lockable. Resilient attachments are categorized into 5 classifications ranging from vertical to universal resiliency. The higher the number of classification, the less torque transferred to the abutment, root or implant.

Based on modes of retention, they are Frictional, Mechanical, Frictional \& Mechanical, Magnetic and Suction types.

Frictional retention is resistance to the relative motion of two or more surfaces in intimate contact with each other. Mechanical retention is resistance to the relative motion of two or more surfaces due to a physical undercut. M agnetic retention is the resistance to movement caused by a 
magnetic body that attracts certain materials by virtue of a surrounding field of force produced by the motion of its atomic electrons and the alignment of its atoms. Magnets do not provide lateral stability and are contraindicated for flat ridges. It is used in limited applications, heat curing will weaken magnets and they are liable to corrode.

Frictional and Mechanical retention combines both features of frictional and mechanical retention as discussed. The Score-PD attachment is a good example.

Suction is a force created by a vacuum that causes a solid object to adhere to a surface. An example would be a wellfitting denture.

Space considerations also are important while deciding the attachment to be used. The space available vertically, bucco-lingually and mesio-distally play a key role in attachment selection.

The vertical space is measured from the tissue to the marginal ridge or from the margin of the abutment to the marginal ridge of the opposing dentition. Use the full length of the attachment, whenever possible, and place it as low as possible without impinging on the tissue.

Buccal-lingual or labial-lingual space is very critical, especially with removable partial dentures. Buccal-lingual or labial-lingual space should be measured accurately to avoid over conto uring the restoration in this dimension.

An additional $1 \mathrm{~mm}$ should be added to the buccal-lingual measurement for metal precision attachments to allow for the casting alloy. It is best to set the teeth prior to the selection of an attachment. This will aid in the size determination and exact position of the attachment.

Mesial-Distal measurements are critical for intracoronal attachments, since a box preparation is required. To avail maximum use, select the largest attachment possible for the space available.

A good way to show how much reduction is necessary is to prepare the study model. It is also helpful for the dentist to have the intracoronal female component available chairside while the abutment is being prepared.

\section{Attachments Available In India}

Ceka preciline[4]

The principle on which the CEKA attachment is based goes back to the year 1958. In those days, the Belgian dental technician Karel Cluytens patented an elastic spring pin that snaps into a conical female component. Both could be incorporated invisibly into the dental prosthesis, thus offering an alternative to the less aesthetic clasp.

CEKA PRECI-LINE meets the highest quality standards. All attachments and accessories are manufactured at the Swiss company HL Technologywhich fully operates in compliance with the European CE standard as regards $M$ edical Devices, and maintains a quality system based on the international ISO 9001/ISO 13485 standard.

\section{RHEIN 83 and RHEIN 83 USA[5]6]}

Smooth head of the sphere along with an elastic cap is amongst the most widely used attachment.Rhein' 83 has been in business since 1983 and today these products have been used throughout the entire world. The commitment of Rhein' 83 with its knowledge and skills continually being enriched by the contributions of dentists and laboratory technicians, is to be able to improve the actual standards and develop new products by means of original projects. The first branch of Rhein 83 is in Italy while Rhein 83 USA is heir branch in United States.

\section{Clinical Cases}

The relevance of attachments in prosthodontics can be assessed from its wide array of uses.

Applications In The Field:

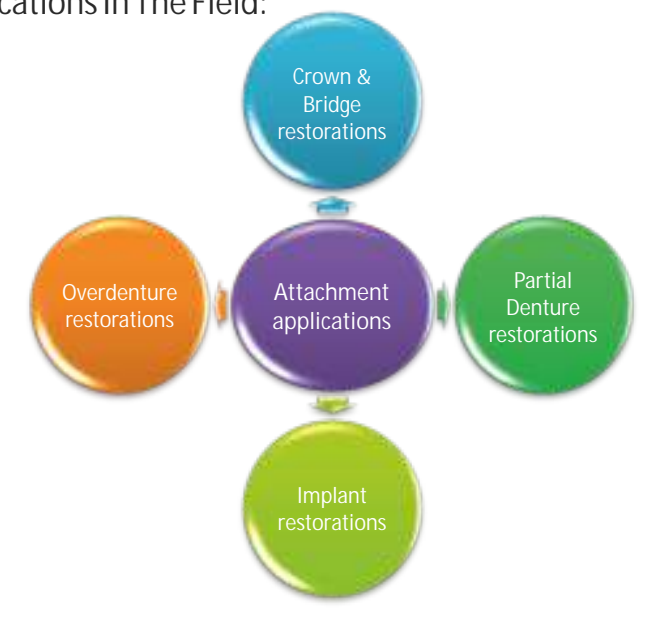




\section{Rehabilitation of Long Span Edentulous Arch Using Custom Made Bar and Ceka Priceline Rider Clips}

The Preci-Horix bar and rider system was developed by Helmut Hader and is the most popular of all bar systems due to its simplicity. The retention of the plastic clips, patient comfort, ease of processing and durability are its assets.

Advantages of the bar system:

1. Easily available female riders in three retention levels to choose from.

2. The bar pattern may be cast in alloy of choice.

3. Suitable for use on implants

4. The soft snap of the rider clip gives reassurance to patients.

The Cekapreciline bars come in a standard size of $1.8 \mathrm{~mm}$ with three types of rider clips, colour coded according to the retention it provides.

White $=$ reduced retention

Yellow $=$ normal retention

Red $=$ increased retention

\section{Case Report 1}

A male patient aged $32 \mathrm{yrs}$., reported to the department of Prosthodontics, complaining of missing front teeth and desired to get them replaced. He gave a history of road traffic accident, a year ago, due to which he sustained several injuries including loss of several anterior teeth. Patient had neither any systemic condition nor prior dental treatment.

On clinical examination, it was noted that patient had a long anterior edentulous span, from right maxillary first premolar to the left maxillary lateral and from the right mandibular second premolar to the left mandibular first premolar. (Fig 1) The remaining teeth had good interception and were periodontally sound. However in the anterior region especially in the mandible, there was hardly any vestibule nor alveolar ridge support after the accident. The patient was unable to accept a removable partial denture and requested for an alternative prosthesis.
The maxillary region had a missing canine on the right side, along with lateral and both central incisors. A fixed partial denture in such a case cannot be designed due to various biomechanical considerations such as the weak abutment on side (1st premolar and lateral incisor), the long edentulousspan and ante'slaw.

Hence, the option of a fixed-removable prosthesis supported by the custom made bar (resembling a header bar) and rider clips was suggested to the patient. The advantages and limitations of the treatment were also addressed before the procedures commenced.

The maxillary right first premolar, left lateral incisor and left canine were prepared as abutments, maintaining as much as parallelism was possible. Similarly, the mandibular left first premolar and right second premolar were prepared to receive metal ceramic crowns. (Fig2) Retraction cords were placed followed by impressions with addition silicone impression materials (Aquasil). Face bow transfer was done.

The casts were retrieved and a mock up arrangement was carried to gauge the position of the teeth on the long edentulous span in order to plan treatment accordingly. (Fig 3) The casts were mounted and wax patterns made on the abutment teeth.

To make the procedures economical, a plastic straw measuring $1.8 \mathrm{~mm}$ in dimension similar to the prefabricated hader bar and of the same form, was used to create the wax pattern which would be replaced by a castable alloy. (Fig 4) The wax pattern of the bar was placed strategically along the crest of the bar to balance the forces from the tongue and lips. Since, the anterior edentulous span is curved, the bar was also fabricated likewise and was connected to the abutment wax patterns with pattern resin.(GC) The bar was attached as cervically as possible without impinging on the alveolar mucosa. The wax patterns were milled so as to ensure parallelism and the path of placement. [7](Fig 5)

Once the wax patterns were cast, milling was done. M etal try in was carried out to ensure marginal integrity, passive 
fit and precise position of the bar. A pick up impression was made of both arches and poured in die stone (Kalrock). The casts were articulated and ceramic was layered over the metal copings. Another trial was done to confirm the shade matching of the ceramic. (Fig 6)

The removable partial denture base was fabricated and artificial teeth arranged. A trial of the waxed denture base was carried out to test esthetics, function and phonetics. (Fig 7) Once it met all criteria and patient satisfaction, the removable denture bases were processed with heat cure acrylic.

The abutment crowns along with the custom made bar were cemented first in the patient's mouth. The rider clips were placed in the centre of the bar and it was picked up into the removable partial denture with help of self cure acrylic. (Fig 8) The prosthesis was cleaned, polished and fitted in. Care and maintenance instructions were given to the patient and recall and review was done.

\section{Management of Unilateral Distal Extension Case Using Semi precision Attachment - Sagix Attachment (Ceca Priceline)}

Preci-sagix is the sagittal ball attachment with segmented female for partial dentures and implant applications. It has a wide range of applications, long lasting retention, patented snap mechanism, easy replacement of parts and is relatively inexpensive.

\section{Case Report 2}

A male patient, age $48 \mathrm{yrs}$, reported to the department of Prosthodontics, complaining of missing posterior teeth and difficulty to chew. Patient had no relevant systemic condition or any prior dental treatment. Intra-oral examination revealed missing right mandibular first and second molar. (Fig9)

Patient was explained the various treatment choice for his clinical situation. The lack of a distal abutment tooth ruled out fixed partial dentures. The patient was neither willing for implant surgical procedures nor was he comfortable with the conventional removable denture. The option of a fixed-removable prosthesis was acceptable by the patient and hence, the procedures were carried out.

The first and second right mandibular premolars were prepared and impressions were made. (Fig 10) Facebow transfer was done and the casts were articulated. Wax patterns were prepared for the premolars.

The ceca Priceline sagix (preci-sagix) comes with 3 components - the castable plastic male, the duplicating dummy rider clip and the female rider clip. The castable male was attached to the wax patterns with the aid of a paralleling mandrill to ensure parallel placement. (Fig 11) This was cast in alloy.

The metal trial was done in patient's mouth and pick up made with the help of addition silicone impression material (Aquasil). Ceramic build up was done for the premolars and the removable partial denture with first and second molar was waxed up and tried in clinically. (Fig 12) The removable partial denture was processed with the duplicating dummy in place of the female rider clip. The splinted premolar crowns, with the sagix attachment, were cemented in the patient's mouth. The removable partial denture was tried for passive fit and then the female rider clip was picked up from the sagix into the partial denture using self cure acrylic in a chairside procedure. (Fig 13)

\section{Discussion}

There are many factors that come into light while considering precision attachments as an integral part of treatment. Although the advent of precision attachments has negated the need for clasps, there are a few drawbacks to this route as well. One main aspect of geriatric patients is the diminished dexterity of the patient, one which often escapes the calculations while designing the prosthesis. Consequently, multiple attachments and complex designing should be avoided. Whenever possible, sticking to the simplest design with the necessary attachment works in good stead. Hence, an attachment should be selected according to the capacity of the patient to maneuver and seat without a third person's help.

Using a laboratory model for the distal extension 


\section{ny)HS}

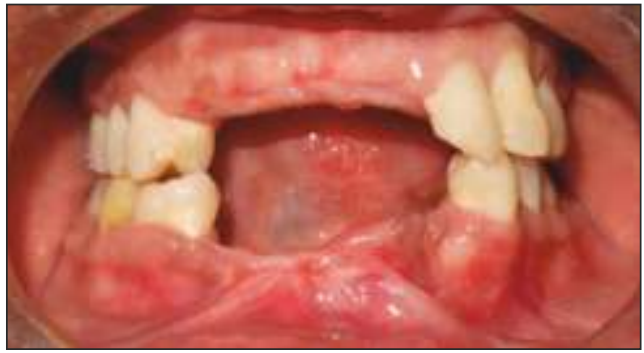

Figure 1 : Pre-operative photograph

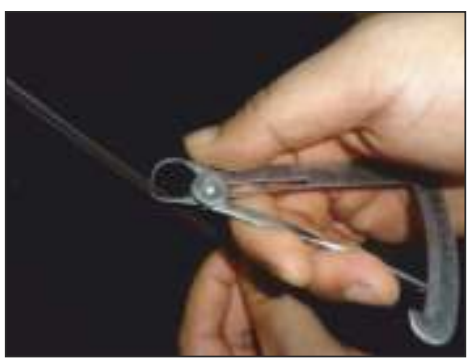

Figure 4 : Dimensions of bar

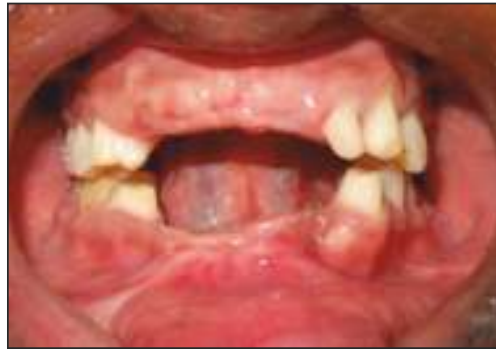

Figure 2 : Preparation of abutments

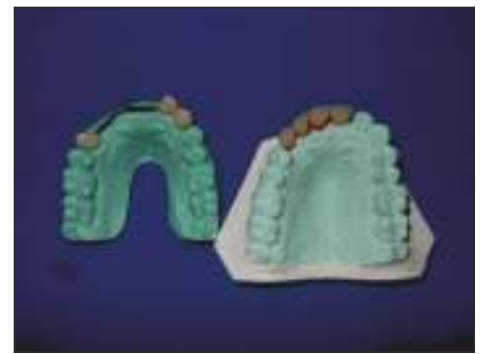

Figure 3 : Wax pattern with custom bar

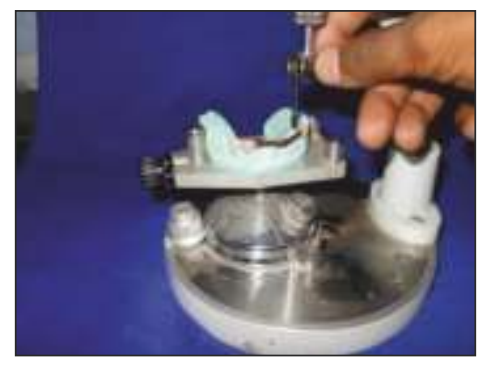

Figure 5 : M illing

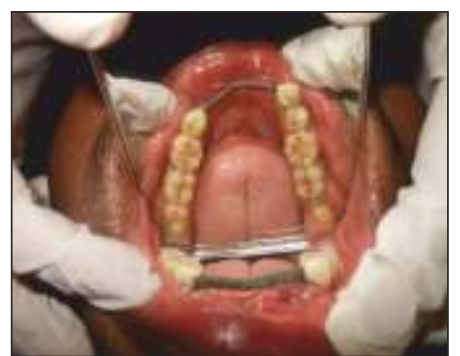

Figure 6 : M etal try in

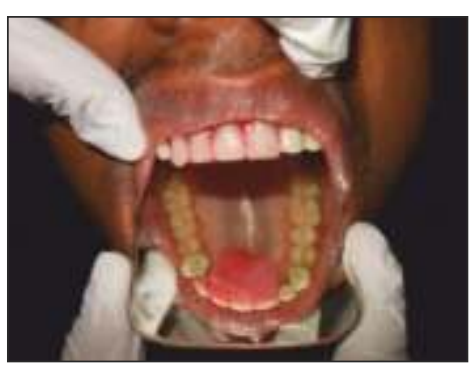

Figure 7 : Try in

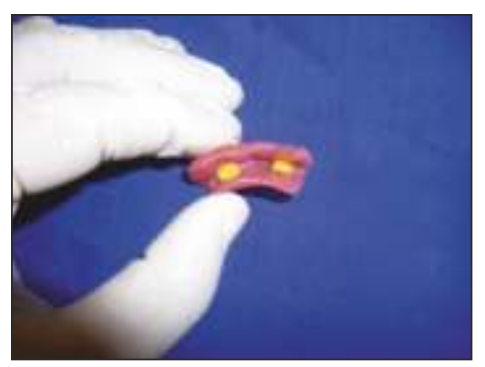

Figure 8 : Pick up of rider clips

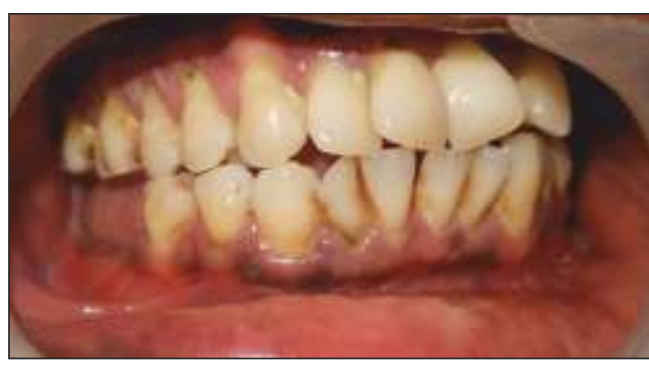

Figure 9 : Pre operative photograph

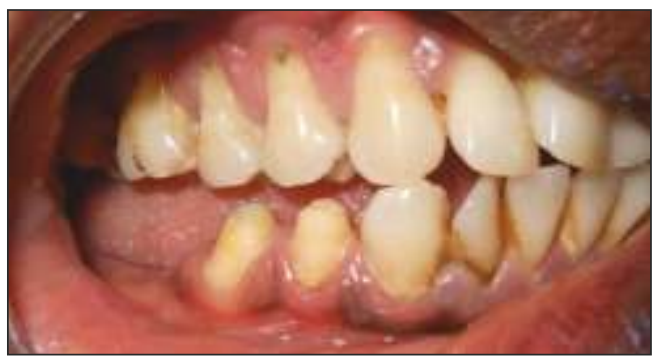

Figure 10 : Tooth preparation

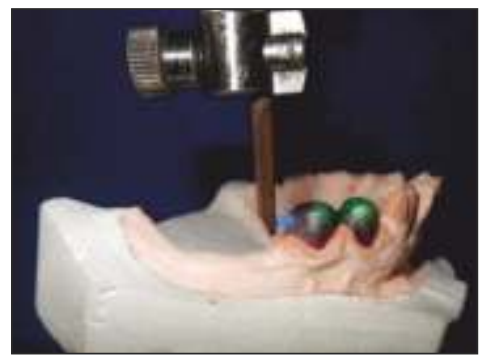

Figure 11 : castable male attache to wax pattern

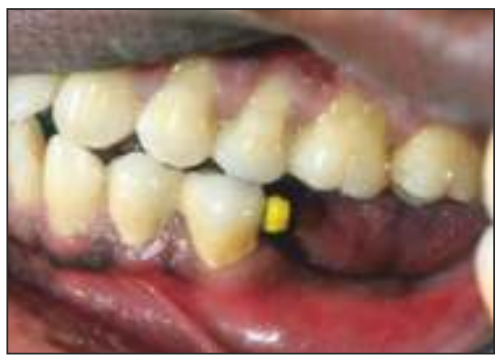

Figure 12 : Try in

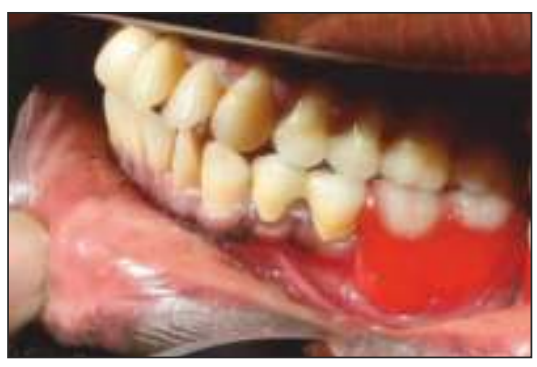

Figure 13 : Trial of denture

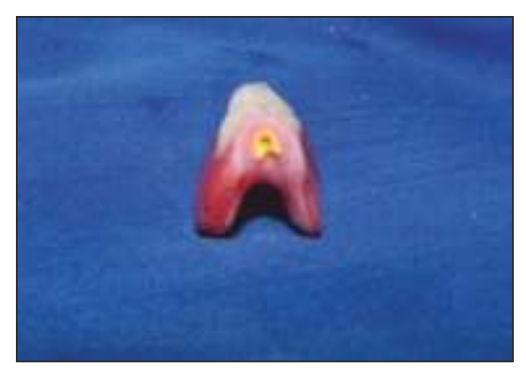

Figure 14 : Pick up of rider clip 
removable partial denture situation, the effect of resilient and rigid precision attachment retainers on abutment tooth and denture base movement was studied. It was found that both abutment tooth and denture base movement was least with the rigid and semi-precision attachments used compared to the resilient attachments.[8] The intra-coronal attachment is suggested to be applied in some cases that the periodontal condition of distal abutment was good, and the extra-coronal attachment is suggested to be used in some cases that the condition of the edentulous alveolar crest is fairly good, while the periodontal condition of the distal abutment was relatively weak.[9]

Another key point to be regarded is the path of insertion of the prosthesis along with attachment. Surveying and milling is imperative during the planning and execution of these prostheses. The casts, wax pattern, metal substructure and the finished prosthesis should be surveyed and milled at every point to ensure a common path of insertion.

Every attachment with the resilient counterpart has a shelf

\section{References}

1. Nigam A, Singh A, Shekhar A, Gupta H. Precision Attachments - An overview. www.journalofdentofacialsciences.com, 2013; 2(4): 41-44

2. Gozneli R, Yildiz C, Vanlioglu B, Evren BA, Kulak-Ozkan Y., "Retention behaviors of different attachment systems: Precious versus nonprecious, precision versus semi-precision, Dent Mater J. 2013;32(5):801-7.

3. H. W. Preiskel, Precision attachments in prosthodontics: The applications of intracoronal and extracoronal attachments,Volume 1. Quintessence Pub. Co., Chicago.1984.

4. "About Us: Ceka preci-line," [Online]. Available: www.cekapreciline.com

5. www.rhein83usa.com.

6. www.rhein83.com. life, given by the manufacturers. The plastic resilient cap undergoes wear from usage and has to be replaced when its retentive capacity is lost. [10] The patient should be informed in advance of this and advised to have regular checkups. The resilient component is easily substituted for a new one without the need to change the entire prosthesis.

The patient must be informed of all treatment options available with the limitations of the same. The cost of the treatment along with its benefits should be gauged by the patient before making a learned choice.

And finally, no attachment is perfect for every application. Every clinical presentation is different and the thorough knowledge of the attachment and its application along with good clinical acumen is required to ascertain the type of attachment that can be used for the patient.

\section{Conclusion}

The use of attachments requires a thorough knowledge of basic prosthodontic principles, appropriate training and experience with the particular attachment used, technical skills and clinical ability and judgment.

7. Brudvik JS1, Shor A, "The milled surface as a precision attachment.," Dent Clin North Am. 2004 Jul;48(3):685-708.

8. Feingold GM, Grant AA, Johnson W, Abutment tooth and base movement witl attachment retained removable partial dentures.J ournal of dentistry,December 1988;16(6):264-268.

9. Wang Y,Mi N,Qing F,Liu F,Chen J, Surface stress analysis of distal extension removable partial denture retained with two types of semiprecision attachments, West China journal of stomatology 2001;19(5):283-286,

10. Hedzelek W,Rzatowski S,Czarnecka B. Evaluation of the retentive characteristics of semi-precision extracoronal attachments.J ournal of Oral Rehabilitation2011;38(6): 462-468. 